\title{
Glutathione Ameliorates Mouse Steatohepatitis
}

\author{
Fengjuan Chen" ${ }^{\# 1}$ Fang Luo ${ }^{\# 2}$, Chunliang Lei ${ }^{1 *}$ and Qingxian Cai ${ }^{2 *}$ \\ ${ }^{1}$ Department of Hepatology, Guangzhou Eighth People's Hospital, Guangzhou, China \\ ${ }^{2}$ Department of Hepatology, The Third People's Hospital of Shenzhen, Shenzhen, China
}

*Corresponding author: Q Cai, Department of Hepatology, The Third People's Hospital of Shenzhen, No 29, Bulan Road, Longgang

district, Shenzhen 518112, China

\section{ARTICLE INFO}

Received: 慧 August 14, 2020

Published: 幽 August 26, 2020

Citation: Fengjuan C, Fang L, Chunliang L, Qingxian C. Glutathione Ameliorates Mouse Steatohepatitis. Biomed J Sci \& Tech Res 29(5)-2020. BJSTR. MS.ID.004851.

Keywords: Reduced glutathione; Steato Hepatitis; Non-Alcoholic Fatty Liver Disease; Non-Alcoholic Fatty Hepatitis; Oxidized Low Density Lipoproteins; Superoxide Radicals

\begin{abstract}
Objectives: Non-alcoholic fatty liver disease (NAFLD) is an increasing epidemic that may promote later stages of liver disease. It was reported that oxidative stress and lipid per oxidation are key pathological factors in non-alcoholic fatty Hepatitis (NASH). The objective of this study was to explore the protective effect of glutathione (GSH) on liver oxidative damage in NASH mouse model.
\end{abstract}

Methods: Male C57BL/6J mice were treated for 30 weeks by a high fat diet and intravenous injections of oxidized low density lipoproteins (oxLDL) without or with daily intra peritoneal injections of GSH for the last 10 weeks. Then the mice were sacrificed with appropriate controls. Blood and liver were harvested for analysis, including plasma and liver triacyl glycerol, total cholesterol, free fatty acids, insulin resistance, malondialdehyde (MDA), GSH, superoxide radicals, protein oxidation, inflammatory markers, and apoptosis in the liver tissue.

Results: The HFD combined with intravenous injections of oxLDL induce NASH in mice, characterized by elevated level of the lipid profiles, MDA, superoxide radical generation, protein carbonyls, and nitro tyrosine and higher expression of markers inflammatory markers, and apoptosis $(\mathrm{p}<0.001)$. Introduction of GSH significantly mitigated these perturbations $(\mathrm{p}<0.001)$.

Conclusions: These experimental findings suggest that intra peritoneal injections of GSH ameliorate liver oxidative damage in NASH mouse model. The results provide a new perspective for understanding NAFLD and increase therapeutic armamentarium of antioxidants.

\section{Background}

Non-alcoholic fatty liver disease (NAFLD) is one of the most common causes of liver disease in western countries and its prevalence has increased globally over the past years [1]. It is estimated that about $25 \%$ of the adults in the United States and more than 30\% individuals in Japan have fatty liver in the absence of excessive ethanol consumption [2]. In China, NAFLD is becoming more and more prevalent and reported to afflict $20 \%$ of the general populations [3]. Despite the alarming rate of NAFLD, there are limited knowledge in the pathogenesis behind NAFLD and no effective medicine for NAFLD approved by governmental agencies.
Therefore, it is urgent to understand the pathogenesis of NAFLD to establish proper treatment [4]. NAFLD is characterized by lipid accumulation in the liver (steatosis) with or without inflammation (hepatitis). The progression from nonalcoholic simple fatty liver (NAFL)to nonalcoholic steatohepatitis (NASH) is a key step in pathogenesis, as it indicates the disease process has been an accelerating stage [5,6]. At present, several mechanisms was found to account for steatohepatitis including increased adipose tissue (VAT), dyslipidemia, gut flora dysfunction, insulin resistance, oxidative stress and lipid peroxidation $[7,8]$. 
At the beginning, the increased VAT and dyslipidemia amplifies the delivery of free fatty acid (FFA) to liver, impairing the hepatic lipid content and initiating the subsequent disease process $[9,10]$. Higher FFA in the liver cause sustain stimulating of neutrophil and macrophage, inducing oxidative stress by the production of reactive oxygen species (ROS) [11]. Furthermore, the metabolism of the excess FFAs may enhance the lipid peroxidation, producing large amount of lipid peroxide (LPO) like Malonaldehyde (MDA) and 4-hydroxynonenal (HNE) [12,13]. Oxidative stress and lipid peroxidation are both important mechanism in the development of NASH. They may bring damage to cell structures such as membranes, proteins and DNA of cell, triggering inflammatory response, leading hepatitis $[14,15]$.

Recent studies have revealed that oxidative stress and lipid peroxidation plays a dominant role in the progression of NAFLD to NASH $[16,17]$. It has been suggested that increased production of superoxide radicals from mitochondria and cytoplasm is crucial for the development of NASH [18]. OxLDL is the highly reactive form of LDLafter lipid peroxidative modification [19]. Until today, a large amount of data has showed oxidized low-density lipoproteins (oxLDL) play an important role inoxidative stress and lipid peroxidation during obesity-related inflammatory disorder, such as metabolic syndrome; type 2 diabetes mellitus and atherosclerosis [20-22]. Recent evidence also suggests oxLDL as a new risk factor for steatohepatitis. OxLDL is capable of activating macrophage, enhancing transmigration of inflammatory cell [23-25] and inducing apoptosis [26]. Administration of oxLDL to HFD-fed mice can built a mouse model for NASH, displaying the entire pathology of NASH, including steatois, hepatic inflammation, oxidative stress and lipid peroxidation [27].

It has been suggested increased oxidative stress correlates with steatohepatitis progression in patients $[28,29]$. Since impaired antioxidant defense mechanisms can partially account for NAFLD pathogenesis, treatment strategies that affect the antioxidant enzymes might be useful for individuals suffering from NAFLD. Reduced glutathione (GSH) is an endogenous antioxidant that protects the liver from different intrinsic and extrinsic oxidants produced as a result of cellular metabolism by neutralizing the oxidants and sustaining cellular homeostasis [30,31]. GSH from GSH hepatocyte transporters is known to show scavenging activity against reactive oxygen species (ROS) generated by Kupffer cells, thereby maintaining the hepatic structural integrity [32-36]. GSH levels are depleted during severe disease caused by enhanced oxidative insult and mitochondrial GSH is believed to act as the first line of defense against damage mediated by superoxide radicals $[35,37]$. However, whether GSH could protect NAFLD has not been well understood. Herein, we study the viability of GSH to combat oxidative stress-mediated steatohepatitis in high fat diet supplemented mice.

\section{Methods}

This study was approved by the Institutional animal ethical committee at the Department of Infectious Diseases, Sun Yat-Sen University, China.

\section{Experimental Animals}

Four-week-old Male C57BL/6J mice purchased from vendors in China were maintained on 12:12 h light and dark cycles and were free access to pellet diet and ad libitum water. After a week of acclimatization, the Non-alcoholic fatty Hepatitis (NASH) mouse model was developed by feeding high fat diet (HFD-60; Oriental Yeast Co. Ltd., Tokyo, Japan) and intravenous injections of oxLDL (Solarbio Co., Ltd. Pekin, China). All mice were divided into three groups. Group I $(\mathrm{n}=10)$ fed a normal diet for 30 weeks. For 30 weeks, with daily intravenous injections of $0.2 \mathrm{ml}$ phosphate buffered saline in the last three weeks and served as the controls. Group II $(n=10)$ received a high fat diet (HFD) along with intravenous injections of oxLDL $0.2 \mathrm{ml}$ on alternate days for 30 weeks. Group III $(n=10)$ received the same experimental regimen as Group II along with daily intraperitoneal injections of GSH for the last 10 weeks (150 mg/kg body weight). The food and water intake of the mice and their body weight was recorded twice a week. During the experimental period, the body weights and organ weights of mice were measured with an electronic balance (ATY 224, Shimadzu, and Columbia, MD). At the end of the experiment, the mice were anaesthetized using diethyl ether and euthanized. Liver tissue was snap frozen by liquid nitrogen immediately and stored at $-80^{\circ} \mathrm{C}$ until further use. Before termination, blood was collected from the retro-orbital sinus using a heparinized syringe. The plasma was separated by centrifugation at $8000 \mathrm{~g}$ and stored at $-80^{\circ} \mathrm{C}$.

\section{Histology}

The liver tissues were fixed, paraffinized, cut to $5 \mu \mathrm{m}$ sections, deparaffinized and subjected for hematoxylin and eosin (H\&E) staining. Histological changes were observed under a light microscope (Olympus Optical Co., Lake Success, NY).

\section{Superoxide Radical Estimation}

The generation of superoxide radicals in the liver was detected using electron spin resonance (ESR)[38]. The brief procedure was as follows:

a) Recorded the ESR spectra on a spectrophotometer (Model UV-2602; Labomed, Inc. USA): an aqueous quartz flat cell (60 $\mathrm{x}$ $10 \times 0.31 \mathrm{~mm}$, effective volume $160 \mu \mathrm{l}$ ) was used.

b) Measured optical absorption spectra: using a multichannel photodetector. A nitrone spin trap 5, 5-dimethyl1-pyrroline N-oxide (DMPO) was used for the detection of superoxide radicals. 
The incubation conditions for the reaction mixture were as follows: mitochondria $0.7-10 \mu \mathrm{g} / \mu \mathrm{l}$ from Groups I, II and III, $70 \mathrm{mM}$ sucrose, $220 \mathrm{mM}$ manitol, 2mM 4-(2-hydroxyethyl)-1piperazineethanesulfonic acid buffer ( $\mathrm{pH} 7.4), 2.5 \mathrm{mM}$ potassium phosphate buffer, $0.5 \mathrm{mM}$ EDTA, $5 \mathrm{mM}$ malate and $10 \mathrm{mM}$ glutamate. ESR was recorded for $2 \mathrm{~min}$ after the addition of the enzyme source and 1.5 M DMPO $(20 \mu \mathrm{l})$ to the reaction mixture. The ESR spectrometer (JES-FA200, JEOL, Tokyo, Japan) parameters were maintained at a magnetic field of $325 \mathrm{Mt}$, power of $1.00 \mathrm{Mw}$, frequency of $94,380 \mathrm{GHz}$, modulation amplitude of $2 \mathrm{Mt}$, gain of 500 , scan time of $0.5 \mathrm{~min}$, scan width of $10 \mathrm{Mt}$, and temperature of $25^{\circ} \mathrm{C}$ throughout the experiment.

\section{TUNEL Assay}

The evaluation of apoptotic cells in tissue sections was performed using TUNEL assay as described by Gavrieli et al. [39]. In brief, tissue sections were subjected to terminal deoxynucleotidyl transferase (TdT)-mediated deoxyuridine triphosphate (dUTP)biotin nick end labeling (TUNEL, Intergen Co., Purchase, NY) according to the manufacturer's instructions.

\section{Western Blot Analysis}

After being subjected to $12 \%$ SDS-PAGE, the samples $(25 \mu \mathrm{g}$ of protein) were transferred onto a nitrocellulose fluoride membrane. The membranes were blocked with 1\% Bovine Serum Albumin in TBST (Tris-buffered saline, $0.01 \%$ Tween 20 ) for $2 \mathrm{~h}$ at room temperature (RT), incubated with the monoclonal rabbit antimouse caspase-3-1:500 (Santa Cruz Biotechnology Inc, Santa Cruz, CA, USA), monoclonal rabbit anti-mouse PARP-1-1:500 (Santa Cruz Biotechnology Inc, Santa Cruz, CA, USA), monoclonal rabbit anti-mouse TNF-a-1:500(Santa Cruz Biotechnology Inc, Santa Cruz, CA, USA), monoclonal rabbit anti-mouse ß-actin-1:500(Santa Cruz Biotechnology Inc, Santa Cruz, CA, USA) antibodies for $2 \mathrm{~h}$ at RT, washed and incubated with goat anti-rabbit HRP-1:1000. The membranes were subjected to enhanced Chem iluminescence reaction and densitometric analyses of the blots were performed using Image J-image analysis software (National Institutes of Health, Bethesda, MD)

\section{Biochemical Analysis}

Aspartateaminotransferase(AST)andAlanineaminotransferase (ALT) activity levels, as well as fasting insulin and glucose levels, were measured by commercial kits (Roche, Manheim, Germany) following manufacturers' instructions. Insulin resistance index was assessed by calculating the homeostasis model assessment (HOMAIR) using the following formula: [fasting plasma glucose ( $\mathrm{mmol} / \mathrm{L}$ ) $\times$ fasting plasma insulin $(\mathrm{mU} / \mathrm{L}) / 22.5]$. Total lipid was extracted from the liver according to Sugawara and Miyazawa [40]. Total cholesterol (TC) (Cholesterol quantitation kit Sigma-Aldrich, St. Louis, MO, USA), triacylglycerol (TG) (Triglycerides Liquicolor Kit, Human, Wiesbaden, Germany), and free fatty acids (FFA) (Wako
NEFA-HR(2) Kit, Sopachem BV, Ochten, the Netherlands) were determined enzymatically using commercial kits according to the manufacturers' protocols. The protein content in the lysates was determined using the Bradford method (Sigma Chemical Co., St. Louis, MO, USA). Further, the levels of malondialdehyde (MDA), $\mathrm{GSH}$, and oxidized glutathione as well as the activities of superoxide dismutase, catalase and glutathione reductase were assayed in the liver tissue of the control and experimental mice. All these assays were carried out using commercial kits according to the protocols provided by the manufacturer.

\section{Statistical Analysis}

Normally distributed data are presented as the mean \pm standard deviation (mean $\pm \mathrm{SD}$ ), skewed data as the median (interquartile range). All data were statistically analyzed by the Statistical Package for Social Sciences (SPSS, Version 16, Chicago, IL). One-way analysis-of-variance (ANOVA) followed by Tukey's multiple comparisons test was performed to compare the results. The results were considered statistically significant when the probability was less than $0.05(\mathrm{p}<0.05)$.

\section{Results}

\section{The Influence of GSH on the Metabolic Index of NASH Mice}

An increase was observed in the body weight of mice in Group II when compared to Group I and Group III mice at 10 weeks $(p<0.05)$ and at the end of the experiment $(p<0.001)$. Further, the liver weights were significantly $(\mathrm{p}<0.05)$ increased in Group II mice (85\%) compared to Group I and Group III mice. We evaluated the glucose and insulin levels in mice from Groups I, II, and III. Fasting glucose levels were significantly higher in Group II mice compared to Group I mice. Additionally, the HOMA-IR levels were increased 8.2 fold in Group II mice compared to mice from Group I $(\mathrm{p}<0.05)$ and there was a significant decrease in HOMA-IR levels in Group III. On the other hand, the plasma glucose and insulin levels were significantly $(\mathrm{p}<0.001)$ increased in Group II mice compared to Groups I and III. However, these perturbations were significantly mitigated in Group III mice compared to Group II mice. We did not observe any significant difference in body weight, glucose and insulin levels HOMA-IR between Groups I and III. The levels of TG, TC, and FFA in the liver tissue were significantly $(p<0.001)$ elevated in Group II mice when compared to Group I mice, whereas, post GSH treatment, these levels were significantly $(\mathrm{p}<0.05)$ decreased. We further estimated the lipid profiles in the plasma. The Group II mice experienced a significant $(\mathrm{p}<0.001)$ increase in their lipid profiles compared to Group I mice. In contrast, these values were significantly recovered post GSH treatment. The activities of AST and ALT were significantly $(\mathrm{p}<0.001)$ elevated in Group II mice when compared to Group I mice. The transaminase activities were significantly lowered in mice from Group III compared to Group II mice. 


\section{Lipid Peroxidation and GSH Levels}

As it was shown that MDA levels were significantly increased in Group II mice compared to Group I mice. This elevation in MDA content was significantly $(\mathrm{p}<0.05)$ lowered by GSH treatment.

\section{The Levels of Superoxide Radicals}

ESR spectroscopy was used for the identification of free radicals We found that the obtained ESR data revealed a rapid increase in the specific signal value in Group II when compared to Group I mice. However, this signal value was significantly decreased in the livers of Group III mice when compared to Group II mice. These findings suggest.

\section{Inflammatory and Apoptotic Markers}

In ordered to investigate the inflammation-mediated hepatic steatosis in high fat diet induced mice, immune blot was performed to calculate the extent of apoptosis and inflammation in liver tissue. Group II showed ( $\mathrm{P}<0.001$ vs. Group I; $\mathrm{P}<0.05$ vs. Group III) pronounced expression levels of active caspase-3, N-terminal cleavage fragment of PARP-1 and TNF-a compared to Group I. The Group III mice exhibited a significant decrease in the expression of caspase-3, PARP-1, and TNF-a compared to Group II mice.

\section{Histopathology}

Histopathological results for the liver tissues from all groups. It can be seen that the livers of Group I mice have normal morphology. Microvesicular and macrovesicular hepatic steatosis around the portal area, with hepatic necrosis, inflammatory cell infiltration and fatty vacuoles. However, all these changes were recovered to normalcy in the livers of Group III mice.

\section{Discussion}

The "two-hit" hypothesis for NAFLD progression suggests that oxidative stress is an important factor in the transition from NAFL to NASH. Oxidized LDL, the lipid peroxidation products of LDL, had been demonstrated to play an important role in metabolic syndrome. But the relation between oxidized LDL and oxidative stress in NASH has not been totally clarified. Holvoet et al. reported that higher oxidized low-density lipoprotein is associated with higher incidence of the metabolic syndrome [41]. Chalasani $\mathrm{N}$ et al. reported that the serum level of ox-LDL was obviously higher in NASH patients than controls [42]. A paper from Veerle Bieghs et al. demonstrated that the anti-oxLDL IgM induced by immunization with pneumococci reduced the steatohepatitis induced by HFD [43]. A paper from Yimin et al. showed that administration of oxLDL to HFD-fed mice successfully found a NASH mice model, of which the clinical and pathological characteristics had not been well clarified [44].

The elevation of lipid peroxidation (MDA) levels can served as typical markers of NASH. Lipid peroxidation is the primary mechanism through which ROS can accentuate the oxidative damage in fatty liver disease $[45,46]$. The condition is responsible for inflammatory signals and thus causes cellular damage. It has been suggested that the accumulation of lipid peroxidation products including 4-hydroxynonenal and MDA causes mitochondrial dysfunction $[47,48]$. This suggestion is supported by Bujanda et al. [48], who reported increased MDA content following NALFD in a rodent model. Given the enormous importance of superoxide radicals in disease biology, we identified the reason for the copious production of superoxides in Group II mice. The ESR data demonstrated a rapid increment in superoxide anions in Group II mice compared to Group I mice. It has been reported that respiratory chain complexes are weakly coupled in NASH settings and serve as ROS producers in addition to increasing mitochondrial fatty acid oxidation $[49,50]$. Increased xanthine oxidase activity and uric acid formation via enhanced purine catabolism are essential to maintain ATP levels, and are also responsible for ROS production in the biological system.

In the current study, the administration of oxLDL to HFD-fed mice successfully induce a NASH mouse model, characterized by characterized by elevated level of the lipid profiles, MDA, superoxide radical generation, protein carbonyls, and nitro tyrosine. This result adds one more evidence to the "two-hit" hypothesis that oxidative stress exerts a strong influence on the progression from NAFL to NASH. Based on the knowledge in the contribution of oxidative stress to hepatic injury in patients with NASH. It is thought that antioxidants may be beneficial to patients with NASH [51]. Recent experimental data has implied that some antioxidants favorably influenced the lipid metabolism and steatohepatitis of NAFLD. Jung TS et al. reported that $\alpha$-lipoic acid reduces hepatic steatosis and inflammatory markers in the liver of ALA-treated rats [52]. A report from $\mathrm{Ma} \mathrm{T}$ et al. suggested that geniposide exerts protective effects against hepatic steatosis in rats fed with a high fat diet by its antioxidant mechanism [53]. Jamil A Haque reported that genetically GSH-deficient mice could be inducing to steatohepatitis by normal diet [54]. Oz HS et al. reported that oral administration of GSH-enhancing agent, the S-adenosyl methionine or 2(RS)n-propylthiazolidine-4(R)-carboxylic acid (PTCA), significantly improved the abnormal enzyme activities, fatty degeneration, inflammation, and necrosis of the NAFLD mouse model induced by methionine-choline deficient (MCD) diet [55].

The present study implied intravenous injections of GSH ameliorated the steatohepatitis in mouse model induced by oxLDL, indicating the antagonistic effect of GSH on oxidative stress and lipid peroxidation of ox-LDL. It is acknowledged that TNF-a plays a vital role in the onset of fatty liver disease-induced liver injury $[56,57]$ and circulating neutrophils affect TNF-mediated caspase-3 activation [58]. PARP-1 cleavage fragments are a hallmark of apoptotic progression [59]. All our observations suggested that the use of GSH down regulate the expression of inflammatory markers and apoptosis factors in the liver tissue, which had been elevated 
by oxLDL. These findings reinforce the hypothesis that GSH may favorably influence the cellular the molecular mechanisms implicated in NAFLD and would be beneficial in the treatment of hepatic steatosis, In conclusion, we demonstrated that GSH treatment can protect mice from NASH-induced oxidative damage. GSH treatment significantly reduced cell death (apoptosis) and TNF-a expression by increasing GSH concentration and inhibiting lipid oxidation. The results suggest that GSH treatment can serve as an effective therapeutic option for NASH.

\section{Footnotes}

Authors' Contribution: Feng-Juan Chen conceived and designed the study. Qing-Xian Cai, Li-Li Wu, Hong Deng and XiaoMan Chen performed the experiments. Qing-Xian Cai and Li-Li Wu wrote the paper, Xiao-Qiong Shao, Qiu-Min Luo reviewed and edited the manuscript. All authors read and approved the manuscript.

Ethical Consideration: zssy [2018] 02-337-01.

Funding Sources: This study was supported by funding from the Medical science and technology projects of Guangzhou city health bureau (2015A011050006) and by grants from the National Natural Science Foundation of China (grant number: 81870597).

\section{References}

1. Raluca Pais, A Sidney Barritt, Yvon Calmus, Olivier Scatton, Thomas Runge, et al. (2016) NAFLD and liver transplantation: Current burden and expected challenges. J Hepatol 65(6): 1245-1257.

2. Estes C, Anstee QM, Arias-Loste MT, Bantel H, Bellentani S, et al. (2018) Modeling NAFLD disease burden in China, France, Germany, Italy, Japan, Spain, United Kingdom, and United States for the period 2016-2030. J Hepatol 69(4): 896-904.

3. Zhu JZ, Zhou QY, Wang YM, Dai YN, Zhu J, et al. (2015) Prevalence of fatty liver disease and the economy in China: A systematic review. World Gastroenterol 21(18): 5695-5706.

4. (2016) European Association for the Study of the Liver (EASL), European Association for the Study of Diabetes (EASD) and European Association for the Study of Obesity (EASO) (2016) EASL-EASD-EASO Clinical Practice Guidelines for the management ofnon-alcoholic fatty liver disease. Diabetologia 59(6): 1121-1140

5. Singh S, Allen AM, Wang Z, Prokop LJ, Murad MH, Loomba R (2015) Fibrosis progression in nonalcoholic fatty liver vs nonalcoholic steatohepatitis: a systematic review and meta-analysis of paired-biopsy studies. Clin Gastroenterol Hepatol 13(4):643-654.

6. Haflidadottir S, Jonasson JG, Norland H (2014) Long-term follow-up and liver-related death rate in patients with non-alcoholic and alcoholic related fatty liver disease. BMC Gastroenterol 14: 166

7. Jing Yuan, Chen Chen, Jinghua Cui, JianXin Wu, Ruifu Yang, et al. (2019) Fatty Liver Disease Caused by High-Alcohol-Producing Klebsiella pneumoniae. Cell Metab 30(4): 675-688.

8. Narasimhan S, Gokulakrishnan K, Sampathkumar R, Farooq S, Ravikumar $\mathrm{R}$, et al. (2010) Oxidative stress is independently associated with nonalcoholic fatty liver disease (NAFLD) in subjects with and without type 2 diabetes. ClinBiochem 43(10-11): 815-821.

9. Chalasani N, Younossi Z, Lavine JE, Diehl AM, Brunt EM, et al. (2012) The diagnosis and management of non-alcoholic fatty liver disease: practice Guideline by the American Association for the Study of Liver Diseases, American College of Gastroenterology, and the American Gastroenterological Association. Hepatology 55: 2005-2023.
10. Paschos P, Paletas K (2009) Non alcoholic fatty liver disease and metabolic syndrome. Hippokratia 13(1): 9-19.

11. Hung YC1, Hong MY, Huang GS (2006) Cholesterol loading augments oxidative stress in macrophages. FEBS Lett 580(3): 849-861.

12. James O, Day C (1999) Non-alcoholic steatohepatitis: another disease of affluence. Lancet 353(9165): 1634-1636.

13. Cabello-Verrugio C, Ruiz-Ortega M, Mosqueira M, Simon F (2016) Oxidative Stress in Disease and Aging: Mechanisms and Therapies. Oxid Med Cell Longev 2016: 8786564.

14. Koek GH, Liedorp PR, Bast A (2011) The role of oxidative stress in nonalcoholic steatohepatitis. Clin Chim Acta 412(15-16): 1297-305.

15. Hung YC, Hong MY, Huang GS (2006) Cholesterol loading augments oxidative stress in macrophages FEBS Lett 580(3): 849-861.

16. Kopprasch S, Pietzsch J, Kuhlisch E, Fuecker K, Temelkova-Kurktschiev T, et al. (2002) In vivo evidence for increased oxidat ion of circulating LDL in impaired glucose tolerance. Diabetes 51(10): 3102-3106.

17. Bourdon E, Loreau N, Blache D (1999) Glucose and free radicals impair the antioxidant properties of serum albumin. FASEB J 13(2): 233-244.

18. Fraley AE, Tsimikas S (2006) Clinical applications of circulating oxidized low-density lipoprotein biomarkers in cardiovascular disease. Curr Opin Lipidol 17(5): 502-509.

19. Ylä-Herttuala S, Palinski W, Rosenfeld ME, Parthasarathy S, Carew TE, et al. (1989) Evidence for the presence of oxidatively modified low density lipoprotein in atherosclerotic lesions of rabbit and man. J Clin Invest 84(4): 1086-1095.

20. Maiolino G, Rossitto G, Caielli P, Bisogni V, Rossi GP, Calò LA (2013) The role of oxidized low-density lipoproteins in atherosclerosis: the myths and the facts. Mediators Inflamm 2013: 714653.

21. Kuppan K, Mohanlal J, Mohammad AM, Babu KA, Sen P, et al. (2019) Elevated serum OxLDL is associated with progression of type 2 Diabetes Mellitus to diabetic retinopathy. Exp Eye Res 186: 107668.

22. Bitorina AV, Oligschlaeger Y, Shiri-Sverdlov R, Theys J (2019) Low profile high value target: The role of OxLDL in cancer. BiochimBiophys Acta Mol Cell Biol Lipids 1864(12): 158518.

23. Stroka KM, Levitan I, Aranda-Espino za H (2012) OxLD L and substrate stiffness promote neutrophil transmigration by enhanced endothelial cell contrac-tility and ICAM-1. J Biomech 45(10): 1828-1834.

24. Sedgwick JB, Hwang YS, Gerbyshak HA, Kita H, Busse WW (2003) Oxidized low-density lipoprotein activates migration and degranulation of human granulocytes. Am J Respir Cell Mol Biol 29(6): 702-709.

25. McMurray HF, Parthasarathy S, Steinb erg D (1993) Oxidative ly modified low density lipoprotein is a chemoattractant for human T lymphocytes. J Clin Invest 92(2): 1004-1008.

26. Takarada S, Imanishi T, Hano T, Nishio I (2003) Oxidized low-density lipoprotein sensitizes human vascular smooth muscle cells to FAS (CD95)-mediated apoptosis. Clin Exp Pharmacol Physiol 30(4): 289294.

27. Yimin, Furumaki H, Matsuoka S, Sakurai T, Kohanawa M, et al. (2012) Novel murine model for non-alcoholic steatohepatitis developed by combination of a high-fat diet and oxidized low-density lipoprotein. Lab Invest 92(2): 265-281.

28. Koruk M, Taysi S, Savas MC, Yilmaz O, Akcay F, Karakok M (2004) Oxidative stress and enzymatic antioxidant status in patients with nonalcoholic steatohepatitis. Ann Clin Lab Sci 34: 57-62.

29. Videla LA (2009) Oxidative stress signaling underlying liver disease and hepatoprotective mechanisms. World J Hepatol 1: 72-78.

30. Herson PS, Ashford ML (1999) Reduced glutathione inhibits beta$\mathrm{NAD}+$-activated non-selective cation currents in the CRI-G1 rat insulinsecreting cell line. J Physiol 514(Pt1): 47-57. 
31. Kosower NS, Kosower EM (1978) The glutathione status of cells. Int Rev Cytol 54: 109-160.

32. Bernstein JE, Henninger DA, Phillips SB (2008) Prevention of druginduced liver toxicity. Gastroenterology 134(4): A822-A822.

33. Cahill A, Stabley GJ, Wang X, HoekJB (1999) Chronic ethanol consumption causes alterations in the structural integrity of mitochondrial DNA in aged rats. Hepatology 30(4): 881-888.

34. Fernandez-Checa JC, Garcia-Ruiz C, Ookhtens M, Kaplowitz N (1991) Impaired uptake of glutathione by hepatic mitochondria from chronic ethanol-fed rats. Tracer kinetic studies in vitro and in vivo and susceptibility to oxidant stress. J Clin Invest 87(2): 397-405.

35. Ishii H (1996) Oxidative stress in alcoholic liver injury. Alcohol Clin Exp Res 20: 162A-167A.

36. Reiniers MJ, van Golen RF, van Gulik TM, Heger M (2014) Reactive oxygen and nitrogen species in steatotic hepatocytes: a molecular perspective on the pathophysiology of ischemia-reperfusion injury in the fatty liver. Antioxid Redox Signal 21(7): 1119-1142.

37. Hirano T, Kaplowitz N, Tsukamoto H, Kamimura S, Fernandez-Checa JC (1992) Hepatic mitochondrial glutathione depletion and progression of experimental alcoholic liver disease in rats. Hepatology 16: 1423-1427.

38. Scheinok S, Leveque P, Sonveaux P, Driesschaert B, Gallez B (2018) Comparison of different methods for measuring the superoxide radical by EPR spectroscopy in buffer, cell lysates and cells. Free Radic Res 52(10): 1182-1196.

39. Gavrieli Y, Sherman Y, Ben-Sasson SA (1992) Identification of programmed cell death in situ via specific labeling of nuclear DNA fragmentation. J Cell Biol 119(3): 493-501.

40. Yashpal Singh, MK Garg, Nikhil Tandon, Raman Kumar Marwaha (2013) A Study of Insulin Resistance by HOMA-IR and its Cut-off Value to Identify Metabolic Syndrome in Urban Indian Adolescents. J Clin Res Pediatr Endocrinol 5(4): 245-251.

41. Holvoet P (2008) Association between circulating oxidized low-density lipoprotein and incidence of the metabolic syndrome. JAMA 299(19): 2287-2293

42. Chalasani N1, Deeg MA, Crabb DW (2004) Systemic levels of lipid peroxidation and its metabolic and dietary correlates in patients with nonalcoholic steatohepatitis.Am J Gastroenterol 99(8): 1497-502.

43. Bieghs V, van Gorp PJ, Walenbergh SM, Gijbels MJ, Verheyen F, et al (2012) Specific immunization strategies against oxidized low-density lipoprotein: a novel way to reduce nonalcoholic teatohepatitis in mice Hepatology 56(3): 894-903.

44. Yimin, Furumaki H, Matsuoka S, Sakurai T, Kohanawa M, et al. (2012) Novel murine model for non-alcoholic steatohepatitis developed by combination of a high-fat diet and oxidized low-density lipoprotein.Lab Invest 92(2): 265-281.

45. Glantzounis GK, Salacinski HJ, Yang W, Davidson BR, Seifalian AM (2005) The contemporary role of antioxidant therapy in attenuating liver ischemia-reperfusion injury: a review. Liver Transpl 11(9): 1031-1047.

\section{ISSN: 2574-1241}

DOI: 10.26717/BJSTR.2020.29.004851

Chunliang Lei, Qingxian Cai. Biomed J Sci \& Tech Res

This work is licensed under Creative

Commons Attribution 4.0 License

Submission Link: https://biomedres.us/submit-manuscript.php
46.Zhang W, Wang M, Xie HY, Zhou L, Meng XQ, et al. (2007) Role of reactive oxygen species in mediating hepatic ischemia-reperfusion injury and its therapeutic applications in liver transplantation. Transplant Proc 39(5): 1332-1337.

47. Albano E (2002) Free radical mechanisms in immune reactions associated with alcoholic liver disease. Free Radic Biol Med 32:110-114.

48. Chawla RK, Watson WH, Eastin CE, Lee EY, Schmidt J, et al. (1998) S-adenosylmethionine deficiency and TNF-alpha in lipopolysaccharideinduced hepatic injury. Am J Physiol 275: G125-G129.

49. Bujanda L, Hijona E, Larzabal M, Beraza M, Aldazabal P, et al. (2008) Resveratrol inhibits nonalcoholic fatty liver disease in rats. BMC Gastroenterol 8:40.

50.Hensley K, Kotake Y, Sang H, Pye QN, Wallis GL, et al. (2000) Dietary choline restriction causes complex I dysfunction and increased $\mathrm{H}(2)$ $\mathrm{O}(2)$ generation in liver mitochondria. Carcinogenesis 21(5): 983-989.

51. Serviddio G, Bellanti F, Sastre J, Vendemiale G, Altomare E (2010) Targeting mitochondria: a new promising approach for the treatment of liver diseases. Curr Med Chem 17(22): 2325-2337.

52. Jung TS, Kim SK, Shin HJ, Jeon BT, Hahm JR, et al. (2012) a-lipoic acid prevents non-alcoholic fatty liver disease in OLETF rats. Liver Int 32(10): 1565-1573.

53. Ma T, Huang C, Zong G, Zha D, Meng X, et al. (2011) Hepatoprotective effects of geniposide in a rat model of nonalcoholic steatohepatitis.J Pharm Pharmacol 63(4): 587-593.

54. Jamil A Haque, Ryan S McMahan, Jean S Campbell, Masami ShimizuAlbergine, Angela M Wilson, et al. (2010) Attenuated progression of diet-induced steatohepatitis in glutathione-deficient mice. Lab Invest. December 90(12): 1704-1717.

55. Oz HS, Im HJ, Chen TS, de Villiers WJ, McClain CJ (2006) Glutathioneenhancing agents protect against steatohepatitis in a dietary model. J Biochem Mol Toxicol 20(1): 39-47.

56.Birk AV, Liu S, Soong Y, Mills W, Singh P, et al. (2013) The mitochondrialtargeted compound SS-31 re-energizes ischemic mitochondria by interacting with cardiolipin. J Am Soc Nephrol 24(8): 1250-1261.

57. Okajima K, Harada N, Kushimoto S, Uchiba M (2002) Role of microthrombus formation in the development of ischemia/reperfusioninduced liver injury in rats. ThrombHaemost 88: 473-480.

58. Geering B, Gurzeler U, Federzoni E, Kaufmann T, Simon HU (2011) A novel TNFR1-triggered apoptosis pathway mediated by class IA PI3Ks in neutrophils. Blood 117(22): 5953-5962.

59. Chaitanya GV, Steven AJ, Babu PP (2010) PARP-1 cleavage fragments: signatures of cell-death proteases in neurodegeneration. Cell Commun Signal 8:31.

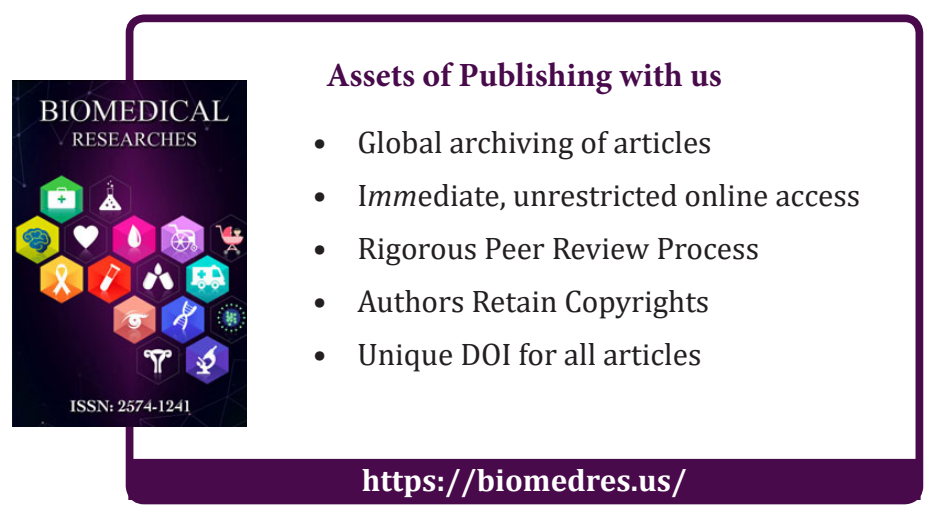

Copyright@ Chunliang Lei, Qingxian Cai | Biomed J Sci \& Tech Res | BJSTR. MS.ID.004851. 